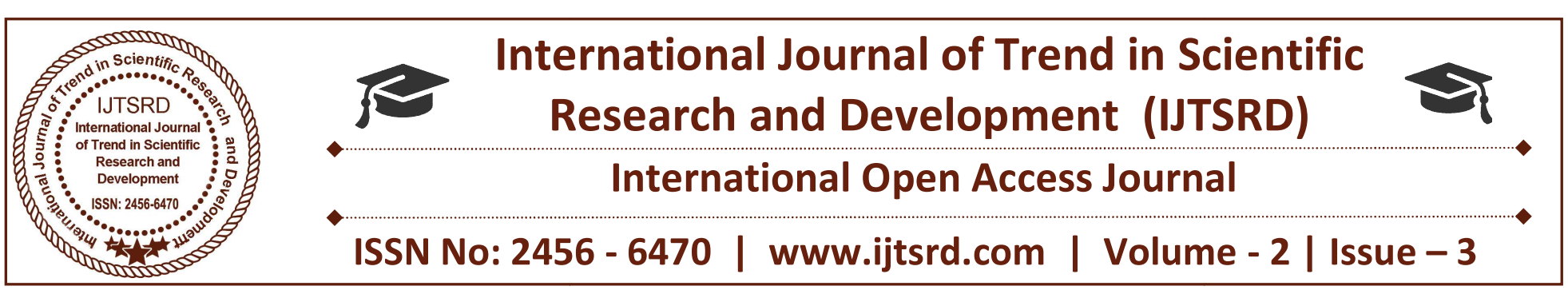

\title{
Multiple Behaviour in Autonomous Robotic Vehicle
}

\author{
Vignesh. S, Santosh. P, Suresh. S \\ Department of CSE, Sri Muthukumaran Institute of Technology, Chennai, Tamil Nadu, India
}

\begin{abstract}
The advancement in mobile robotics in recent decades have inspired and cemented a belief that multiple autonomous robotic agents, often cooperatively helping the humans. In order to provide more generalized adaptive capability in dynamic environments, it is desirable to exclude as many detail assumptions as possible. In order to utilize autonomous mobile robots in real life, the ability of adapting themselves to the environment which change in determinately. Studies have been conducted applying diverse algorithms to robot control for the learning of motion rules and path planning in general environments. . Utilizing DNA programming, Kozza showed that robots can find out motion rules for roaming around grid spaces to get preys, with environmental information only. The robot's sensor information and position data relative to the obstacle is entered, classified to generate representative pattern, which in turn is entered into the Associative Memory to generate generalized motion rules. The desired destination can be determined with information the robot generates.
\end{abstract}

Keywords: Arduino microcontroller, BO motor, motor shield, relay, sensors ,ultrasonic

\section{INTRODUCTION}

The autonomous driving has been received considerable attention from the robotics community. Countless excellent autonomous vehicles have proved their extensive application prospect in the historical events. The main aim of this paper is to represent a modified method in path planning along with decision making based on our algorithm [1]. As two key parts of an autonomous vehicle system, path planning and decision making directly affect the driving behaviour of an autonomous
Vehicle. So nearly all the robot researchers have spent great efforts on them since the first appearance of robots. "Baseline" system that used implementations of $D *$ for global planning based on stereo vision to serve in the unstructured environment. They conduct path planning and decision making in an obstacle map made through vision SLAM, which is flexible and accurate. Because the robot is small and its speed is relatively slow, low-frequency planning speed $(1 \mathrm{~Hz})$ and Studies have been conducted based on applying diverse algorithms to control robot for the learning about motion rules and planning the path in generalized fic environments. Utilizing DNA programming, Kozza [1] showed that robots can find out motion rules for roaming around grid spaces to get preys, with environmental information only. In Difference to the conventional robots that carry out passive and also repetitive motions, while the autonomous mobile robots can recognize all the changes that take place in environments of work, and be able to judge the situations autonomously, also it can move or interact with human autonomously. In order to utilize or implement the autonomous mobile robots in real life, the ability to adapt themselves to all the environments which change indeterminately. Also this end studies have been conducted mainly for the development of robots which can be able to adapt themselves to given environments at any time and also without environment specific knowledge of explicit external control or without defining it. Hence in order to provide a more generalized adaptive capability in the dynamic environments, it's desirable to exclude as many detailed assumptions as possible. Taking all the many prior conditions in which may occur among the real environments into consideration, the adaptive ability will become restricted and also in will result in losing universality. Therefore, the applicability will make it difficult to get verified, and 
also even make it applicable; its usefulness will be restricted to specific environment, making it complex to perform desired capabilities when the environments change.

\section{ARCHITECTURE}

The prior knowledge that a robot will have for a new environment is completely zero. It has only the basic motion rules that are entered by the user, therefore, the situations that which the robot can adapt itself is very limited. In this paper, the Sub Sumption-like [3] [4] architecture as shown in Fig. I is used. Using the sensors information, the linear as well as angular velocities of the robot is being controlled with accordance to the different types of situations to avoid obstacles, and to approach the goal. The algorithm for the motion determination is being constituted with IFTHEN method that has the advantage of judgment by deciding the simple comparative operations without a large amount of volume in calculations.

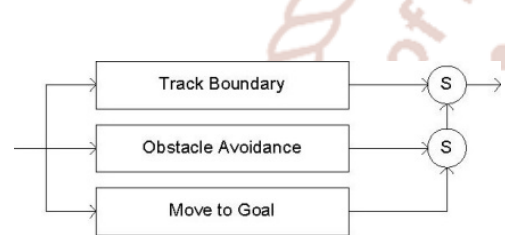

Fig. I Sub Sumption - like Architecture
By applying more no of restrictions as well as assumptions, it is much more applicable to the current diverse situations. Therefore the results of simulation of this study conducted using the basic motion rules [6] is been shown in Fig. 2. The robot's motion is to be available in virtual environments only for the defined sensor information which have multiple obstacles. However, the 'trap phenomenon' which makes the endless loop at the same location occurs at the well-shaped obstacles. By generating a welldefined sub-goal with addition to the preset goal or by using the path use method this can be easily overcome.
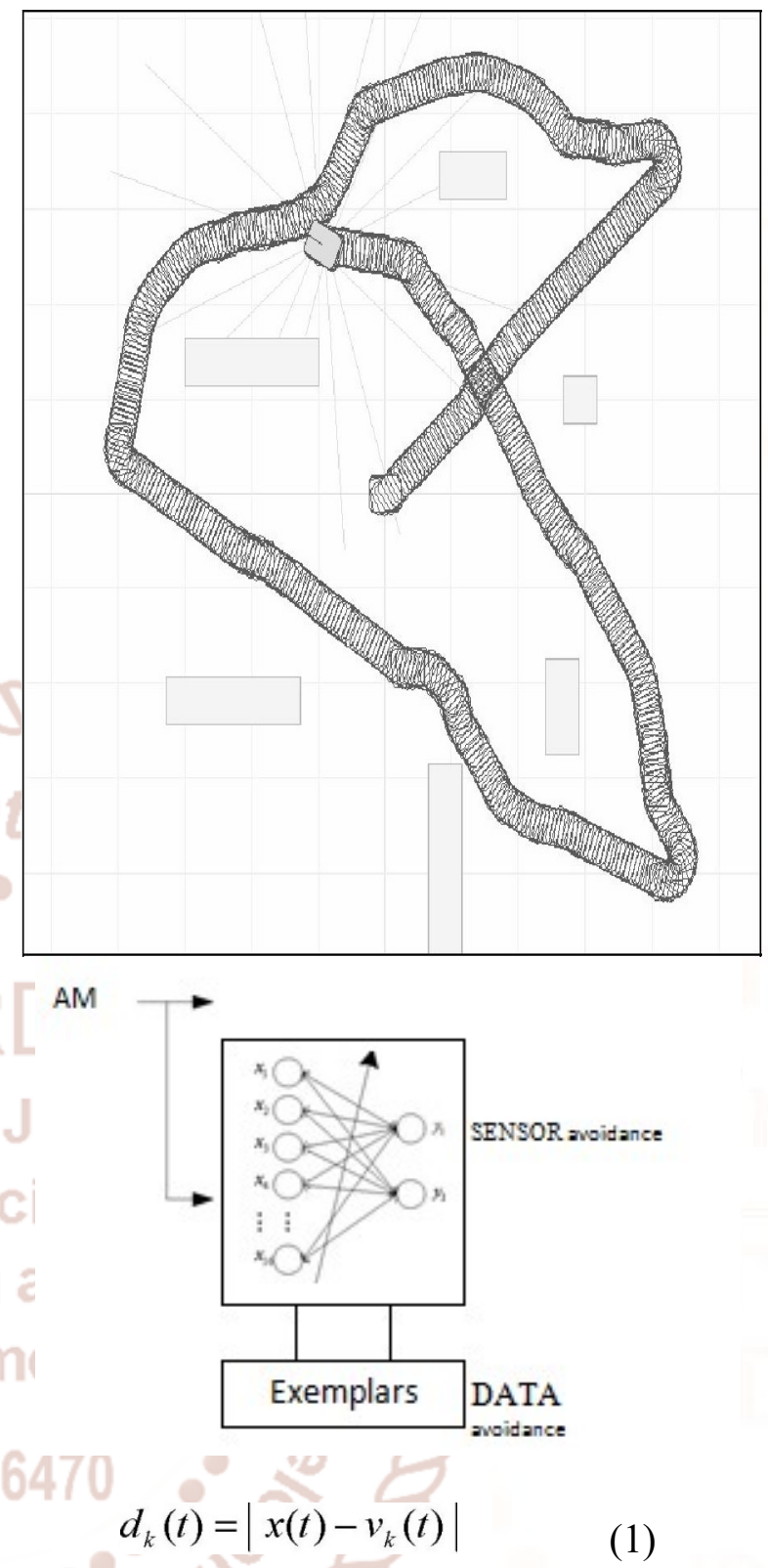

Therefore similar patterns are recognized and also the sub-goal is generated using AM if dk (i) is less than a certain distance, the updated using the equation (2) is done if there is a most similar representative pattern $\mathrm{v}$ (i)

$$
v(t+l)=\quad e \quad x(t)-v(t)
$$

Is the learning rule, the value of which is 0.1 in this paper as obtained by experiments. Value of the similarity $\mathrm{dk}(\mathrm{t})$ is 0.2 .

\section{A. Associative Memory}

Among neural network Associative Memory (AM) is a kind, comprised of input and output layers. Each layer is completely connected followed by the next layer, but there is no connection that exists between the neurons [7] among same layer. AM makes use of one-shot-training method which does not modify weight value during operation but as determined at 
initial stage. Learning data determines weight value, and the associated vector is outputted potentially, when the input vector is applied. The vector is taken as an input at opposite direction and the potentially associated vector is generated in the back position of the input layer. Resonance can only be generated if the final vector is same as originally entered vector. Basically the $\mathrm{k}$ pairs of learning data is expressed with (xk, YK). Hence in order to determine weight matrix, Weight matrix $\mathrm{W}$ and transposition matrix $\mathrm{W}$ can be obtained by following equation

$$
\begin{gathered}
W=X_{1}^{T} Y_{1}+X_{2}^{T} Y_{2}+\ldots+X_{\llbracket}^{T} Y_{\llbracket} \\
W^{T}=Y_{\imath}^{T} X_{\imath}+Y_{\imath}^{T} X_{\imath}+\ldots+Y_{\imath}^{T} X
\end{gathered}
$$

The AM used in this paper has the input layer of 16 sensor information and outputs, as the output layer, the relative distance and angle of the sub-goal in order for the robot to avoid the obstacle in accordance with the location of the obstacle.

Actually, there won't be any sort of representative pattern in the initial stage, the weight matrix of AM is initialized to be zero and obtained by using the representative pattern generated as in section 2. 2. Fig. 3 in this paper the architecture is illustrated.

\section{A. Abbreviations and Acronyms}

DTMF- Dual Tone Multiple Access, IR- Infra Red, BO- Battery Operation, DC- Direct Current, USBUniversal Serial Bus.

\section{COMPONENTS}

\section{A. ARDUINO MICROCONTROLLER}

The Arduino Uno is a microcontroller board based on the ATmega328 (datasheet).it has 14 digital input/output pins(of which 6 can be used as PWM outputs), 6 analog inputs, a $16 \mathrm{M} \mathrm{Hz}$ crystal oscillator, a USB connection, a power jack, an ICSP header, and a reset button. It contains everything needed to support the microcontroller; simply connect it to a computer with a USB cable or power it with an ACto-DC adapter or battery to get started. The Uno differs from all preceding boards in that it does not use the FTDI USB-to-serial driver chip. Instead, it features the Atmega8U2 programmed as a USB-toserial converter. "Uno" means one in Italian and is named to mark the upcoming release of Arduino 1.0. The Uno and version 1.0 will be the reference versions of Arduino, moving forward. The Uno is the latest in a series of USB Arduino boards, and the reference model for the Arduino platform. The operating voltage is $5 \mathrm{~V}$ and its input voltage is

\section{Arduino UNO}
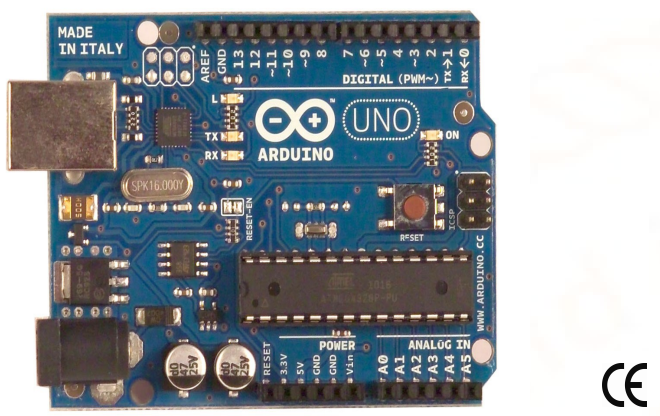

(recommended) 7-12V, The digital I/O Pins has 14 out of which 6 provide PWM output and Analog Input Pins is 6 in no also the DC current per $\mathrm{I} / \mathrm{O}$ pin is 40 $\mathrm{mA}$ and the Flash Memory is $32 \mathrm{~kb}$ out of which 0.5 $\mathrm{kb}$ used by boot loader and SRAM is $2 \mathrm{~kb}$ and EEPROM is $1 \mathrm{~kb}$ and the clock speed of the board is $16 \mathrm{MHz}$.The Arduino Uno has a number of facilities for communicating with a computer, another Arduino,or other microcontrollers. The ATMEGA328 provide UART TTL $(5 \mathrm{~V})$ serial communication ,which is available digital pins 0 (RX) and 1 (TX). An ATmega8U2 on the board channels this serial communication over USB and appears as a virtual com port to software on the computer. The ' $8 \mathrm{U} 2$ firmware uses the standard USB COM drivers, and no external driver is needed. However, on Windows, an *.inf file is required. The Arduino software includes a serial monitor which allows simple textual data to be sent to and from the Arduino board. The RX and TX LEDs on the board will flash when data is being transmitted via the USB-to serial chip and USB connection to the computer (but not for serial communication on pins 0 and 1 ).

\section{B. ULTRASONIC SENSOR}

The HC-SR04 Ultrasonic Module has 4 pins, Ground, VCC, Trig and Echo. The Ground and the VCC pins of the module needs to be connected to the Ground and the 5 volts pins on the Arduino Board respectively and the trig and echo pins to any Digital $\mathrm{I} / \mathrm{O}$ pin on the Arduino Board. 


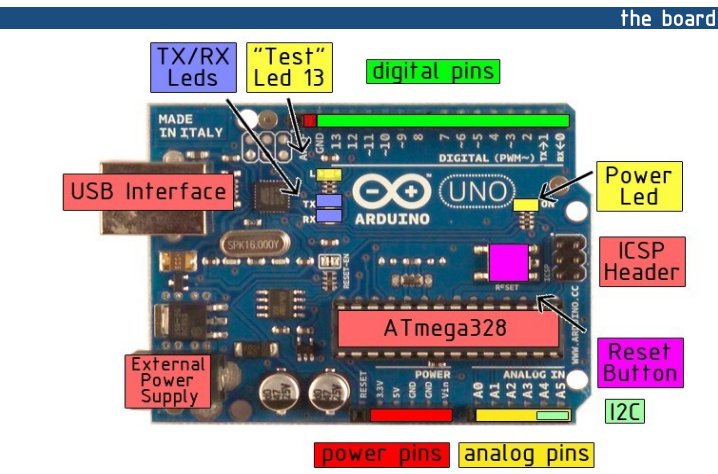

In order to generate the ultrasound you need to set the Trig on a High State for $10 \mu \mathrm{s}$. That will send out an 8 cycle sonic burst which will travel at the speed sound and it will be received in the Echo pin. The Echo pin will output the time in microseconds the sound wave travelled.

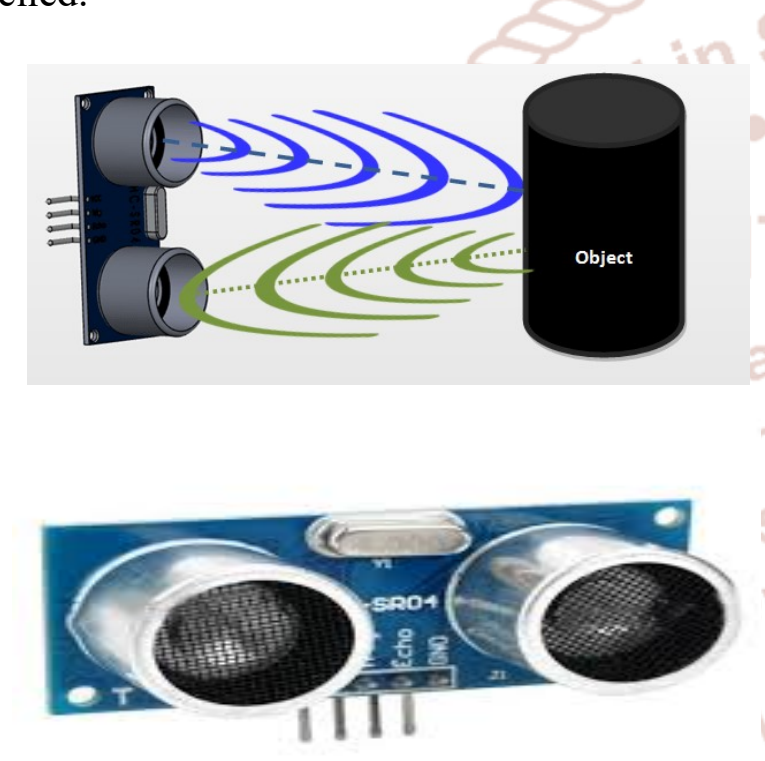

It emits an ultrasound at $40000 \mathrm{~Hz}$ which travels through the air and if there is an object or obstacle on its path It will bounce back to the module. Considering the travel time and the speed of the sound you can calculate the distance.

\section{SG 90 9b MICRO SERVO}

Tiny and lightweight with high output power. Servo can rotate approximately 180 degrees (90 in each direction), and works just like the standard kinds but smaller. You can use any servo code, hardware or library to control these servos. Good for beginners who want to make stuff move without building a motor controller with feedback \& gear box, especially since it will fit in small places. It comes with a 3 horns (arms) and hardware. Its weight is $9 \mathrm{~g}$ and dimension is $22.2 \times 11.8 \times 31 \mathrm{~mm}$ approx. along with it the Stall torque is around1.8, the Operating speed is
$0.1 \mathrm{~s} / 60$ degree and its voltage is $4.8 \mathrm{~V}(\sim 5 \mathrm{~V})$, dead band width is $10 \mu$ s temperature range: $0{ }^{\circ} \mathrm{C}-55^{\circ} \mathrm{C}$

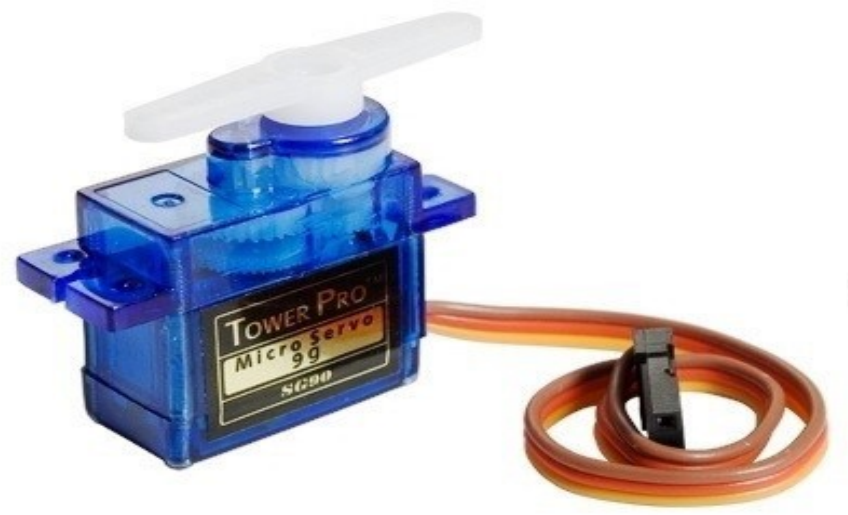

\section{OTHER COMPONENTS ARE}

Arduino Motor Shield, Analog and Digital IR sensor, HC-SR0 4 Base Module, DTMF module, BO Motor L Type, 65X30 Rubber Wheel, Metallic Castor Wheel, ARK-01 ,Wooden Chassis, 6XAA Battery Holder, F2F Jumper Wires, USB A to B Cable, $3.5 \mathrm{~mm}$ Aux. Cable, Castor Screw Nut set,

Motor Screw Nut set, Battery Holder Nut Screw Set, IR sensor Screw Nut set, Arduino Screw Stud Set, DTMF Screw Stud Set,

\section{PROGRAMMING}

void setup () \{

pinMode( dataPin, OUTPUT); // Setting up the motor shield.

pinMode(latchPin, OUTPUT);

pinMode(clockPin, OUTPUT);

pinMode(en, OUTPUT);

digitalWrite(en, LOW);

forward(); // This funtion for forward robot motion \}

\section{CONCLUSION}

Obstacle avoidance algorithm for vehicular robot is discussed in this paper. Since this robot depends heavily On ultrasonic sensor which is also discussed well in this Paper. Destination validation is also a key thing in our Project. We hope that survey would serve to guide the Design and implementation of future bot system that are Not only secure, but scalable and usable in dynamic environment. 


\section{REFERENCES}

1. E. Abele, M. Dervisopoulos, and B. Kuhrke, "Bedeutung und Anwendung von Lebenszyklusanalysen bei Werkzeugmaschinen," in Lebenszykluskosten Optimieren: Paradigmenwechsel für Anbieter und Nutzer von Investitionsgütern, S. Schweiger, Ed. Wiesbaden, Germany: Gabler, 2009, pp. 51-80.

2. R. Isermann, Fault-Diagnosis Systems: An Introduction From Fault Detection to Fault Tolerance. Berlin, Germany: Springer-Verlag, 2006.

3. R. Isermann, Fault-Diagnosis Applications: Model-Based Condition Monitoring: Actuators, Drives, Machinery, Plants, Sensors, and FaultTolerant Systems. Berlin, Germany: Springer-Verlag, 2011.

4. K. Toussaint, N. Pouliot, and S. Montambault, "Transmission line maintenance robots capable of crossingobstacles: State-of-the-art review and challenges ahead,"J. Field Robot., vol. 26, no. 5, pp. 477-499, 2009.

5. P. Debenest et al., "Expliner-Robot for inspection of transmission lines," in Proc. IEEE Int. Conf. Robot. Autom., May 2008,

6. C. Birkenhofer, K. Regenstein, J. M. Zöllner, and R. Dillmann, "Architecture of multi-segmented inspection robot KAIRO-II," inRobot Motion Control. London, U.K.: Springer, 2007, pp. 381389.

7. L. Pfotzer, S. Ruehl, G. Heppner, A. Roennau, and R. Dillmann, "KAIRO 3: A modular reconfigurablerobot for search and rescue field missions," inProc. IEEE Int. Conf. Robot. Biometrics, Dec. 2014, pp. 205-210.

8. X. Gao et al., "Multifunctional robot to maintain boiler water-cooling tubes," Robotica, vol. 27, no. 6, pp. 941-948, 2009.

9. G. Paul, S. Webb, D. Liu, and G. Dissanayake, "Autonomous robot manipulator-based exploration and mapping system for bridge maintenance,'Robot. Autonomous Syst., vol. 59, nos. 7-8, pp. 543-554, 2011.

10. G. Paul, P. Quin, C.-H. Yang, and D. Liu, "Key feature-based approach for efficient exploration of structured environments," in Proc. IEEE Int. Conf. Robot. Biomimetics, Dec. 2015, pp. 90-95. 